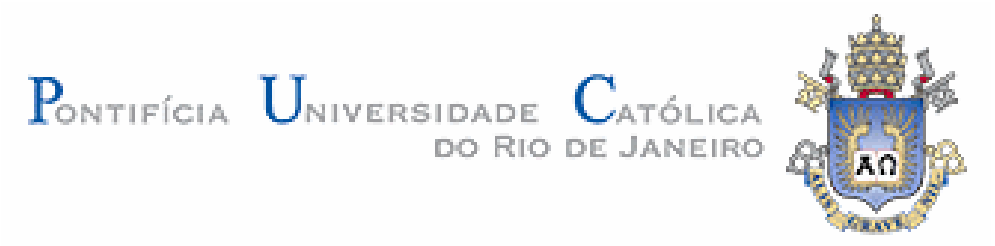

Renata de Albuquerque de Azevedo

\author{
Qualificação profissional de trabalhadores \\ na indústria de óleo, gás e energia \\ na região Nordeste do Brasil
}

Dissertação de Mestrado (Opção profissional)

Dissertação apresentada como requisito parcial para obtenção do título de Mestre pelo Programa de Pós-Graduação em Administração da PUC-Rio.

Orientadora: Sandra Regina da Rocha-Pinto

Rio de Janeiro, julho de 2009 


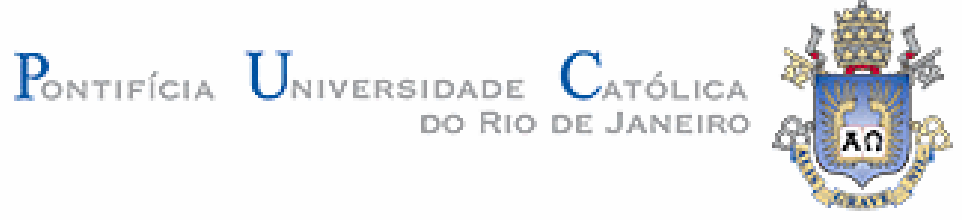

Renata de Albuquerque de Azevedo

\section{Qualificação profissional de trabalhadores \\ na indústria de óleo, gás e energia na região Nordeste do Brasil}

Dissertação apresentada como requisito parcial para obtenção do título de Mestre pelo Programa de PósGraduação em Administração da PUC-Rio. Aprovada pela Comissão Examinadora abaixo assinada.

Profa. Sandra Regina da Rocha-Pinto Orientadora

Departamento de Administração - PUC-Rio

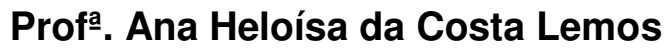
Departamento de Administração - PUC-Rio

Profa. Maria Teresa Correia Coutinho FACC - UFRJ

Prof. Nizar Messari Vice-Decano de Pós-Graduação do CCS

Rio de Janeiro, 13 de julho de 2009 
Todos os direitos reservados. É proibida a reprodução total ou parcial do trabalho sem autorização da universidade, da autora e da orientadora.

\section{Renata de Albuquerque de Azevedo}

Graduou-se em Engenharia de Produção Elétrica, na Pontifícia Universidade Católica do Rio de Janeiro, em 2000, tendo feito pós-graduação em Gestão Empresarial, na Fundação Getúlio Vargas do Rio de Janeiro, em 2003. Possui a Certificação Internacional em Gerenciamento de Projetos PMP (Project Management Professional) pelo PMI (Project Management Institute) e a Certificação como Auditor Líder da Qualidade pelo IRCA (International Register of Certificated Auditors). No âmbito profissional, trabalhou na instituição financeira Losango Promoções de Vendas por cerca de quatro anos, e desde 2003, trabalha como consultora na Accenture do Brasil Ltda.

Ficha Catalográfica

Azevedo, Renata de Albuquerque de

Qualificação profissional de trabalhadores na indústria de óleo, gás e energia na região Nordeste do Brasil / Renata de Albuquerque de Azevedo; orientadora: Sandra Regina da Rocha-Pinto. - 2009.

116 f. : il. (color.) ; $30 \mathrm{~cm}$

Dissertação (Mestrado em Administração)-Pontifícia Universidade Católica do Rio de Janeiro, Rio de Janeiro, 2009.

Inclui bibliografia

1. Administração - Teses. 2. Qualificação profissional. 3. Treinamento. 4. Indústria brasileira de óleo. 5. Gás e energia. 6. Estudo de caso. I. Rocha-Pinto, Sandra Regina da. II. Pontifícia Universidade Católica do Rio de Janeiro. Departamento de Administração. III. Título. 
Aos meus pais Luiz Flavio (in memorian) e Lucia, pelo apoio, dedicação e exemplo de vida.

Ao meu marido Mauro e à minha filha Luisa, pelo amor e carinho. 


\section{Agradecimentos}

Aos meus pais, Luiz Flavio (in memorian) e Lucia, pela educação recebida e força constante em buscar meu crescimento pessoal e profissional.

Ao meu marido, Mauro, pelo apoio e compreensão.

À minha irmã, Patricia, pela amizade.

À minha orientadora, Prof ${ }^{a}$ Sandra Regina da Rocha-Pinto, pela motivação, incentivo, direção e ajuda em permanecer no caminho certo para conclusão desta pesquisa.

Aos professores que aceitaram participar da Comissão Examinadora, pelas sugestões apresentadas e colaboração para conclusão da dissertação.

Aos profissionais que me ajudaram a conseguir os contatos dos entrevistados para realização da pesquisa de campo, em especial, Victor Saldanha, Antônio Sotero e Danúbio Saraiva.

A todos os profissionais que participaram e colaboraram para o desenvolvimento deste estudo com suas entrevistas, perspectivas, experiências, depoimentos e opiniões.

A todos os professores do Departamento, que nos transmitiram de forma muito valiosa os conhecimentos deste curso de mestrado.

Aos amigos e aos colegas do curso de mestrado da PUC-Rio, especialmente, Paulo Roberto Maisonnave, Camila Drubscky, Renata Coutinho, Paola Parigot, Ticiana Neves e Suzana Duprat, pela amizade, motivação e colaboração.

Aos funcionários do IAG, que sempre ajudaram durante todo o curso de mestrado. 


\section{Resumo}

Azevedo, Renata de Albuquerque de; Rocha-Pinto, Sandra Regina da. Qualificação profissional de trabalhadores na indústria de óleo, gás e energia na região Nordeste do Brasil. Rio de Janeiro, 2009. 116p. Dissertação de Mestrado (Opção profissional) - Departamento de Administração, Pontifícia Universidade Católica do Rio de Janeiro.

A indústria de óleo, gás e energia, especificamente, se tratando da Petrobras, vem apresentando, nos últimos anos, investimentos crescentes em projetos e empreendimentos, de forma a aumentar sua produção e se tornar mais competitiva nos cenários nacional e mundial. Com efeito, o objetivo principal deste presente estudo é identificar, diante a perspectiva dos gestores de empresas que realizam obras para essa indústria, se a mão-de-obra disponível na região Nordeste do Brasil está qualificada para atender às demandas para construção de projetos e empreendimentos, e identificar, a partir das percepções dos entrevistados, as ações que vem sendo desenvolvidas para contornar as situações de falta de mão-de-obra qualificada nessa região. A metodologia de pesquisa foi desenvolvida a partir de uma abordagem qualitativa, utilizando o método de estudo de caso. A interpretação e a análise dos resultados obtidos na pesquisa basearam-se no referencial teórico, fundamentado na revisão da literatura pertinente ao tema da pesquisa. Como resultados do estudo, quatro categorias temáticas emergiram da análise das entrevistas: a percepção da qualificação profissional dos trabalhadores; o treinamento para a qualificação profissional; crescimento e oportunidades da indústria de óleo, gás e energia na região Nordeste; e alinhamento e parceria para a melhoria da qualificação dos profissionais. Contudo, apesar das dificuldades apresentadas quanto à disponibilidade de profissionais qualificados na região estudada, as oportunidades de trabalho existem, e ações estão sendo realizadas com o objetivo de melhorar a qualificação de trabalhadores na indústria de óleo, gás e energia na região Nordeste do Brasil.

\section{Palavras-chave}

Qualificação profissional; Treinamento; Indústria brasileira de óleo, gás e energia; Estudo de caso. 


\section{Abstract}

Azevedo, Renata de Albuquerque de; Rocha-Pinto, Sandra Regina da (Advisor). Professional qualification of workers in the oil, gas and energy industry in Northeastern Brazil. Rio de Janeiro, 2009. 116p. MSc. Dissertation - Departamento de Administração, Pontifícia Universidade Católica do Rio de Janeiro.

The oil, gas and energy industry, specifically in relation to Petrobras, has been presenting increasing investments in projects and enterprises as to increase its production and become more competitive in the national and global scenarios. Thus, the main purpose of this dissertation is to identify the perspective of managers of companies which develop construction work for this relevant industry, whether the workforce available in Northeastern Brazil is qualified to supply the construction of projects and enterprises, and also identify, as from the perspective of the interviewed managers, the actions that have been undertaken to overcome the situations in which there is a lack of qualified workforce in the Region. The research methodology selected comprised quantitative approach following a case study method. The interpretation and analysis of the results obtained in the research were based on the theoretical referential, based on the revision of the applicable doctrine on the matter at hand. As a result of the study, four categories emerged from the analysis of the interviews: the perception of the workers' professional qualification; the training for the professional qualification; growth and opportunities in the oil, gas and energy of the Northeast Region; the alignment and partnership to improve the qualification of the workforce. However, despite the difficulties presented in the availability of qualified professionals, job opportunities exist, and actions are being undertaken in order to improve the qualification of workers in the oil, gas and energy in the Northeast region of Brazil.

\section{Keywords}

Professional qualification; Training; Brazilian oil, gas and energy industry; Case study. 


\section{Sumário}

1 Descrição do Problema 13

1.1. Introdução 13

1.1.1. Prominp 17

1.1.2. PlanSeQ Petróleo e Gás 22

1.2. O Problema de Pesquisa 23

1.3. Objetivos da Pesquisa 24

1.4. Premissas 25

1.5. Delimitação do Estudo 25

1.6. Relevância do Estudo 26

$\begin{array}{ll}\text { 1.7. Estrutura da Dissertação } & 27\end{array}$

2 Referencial Teórico $\quad 28$

2.1. Qualificação Profissional 28

2.1.1. Conceituação sobre a Qualificação Profissional 28

2.1.2. A qualificação no ambiente de trabalho 32

2.1.3. Aspectos políticos, sociais e econômicos da qualificação profissional 34

2.2. Treinamento, Desenvolvimento e Educação 38

2.2.1. Conceituação 38

2.2.2. A participação das empresas em treinamento, desenvolvimento e educação

3 Metodologia do Estudo $\quad 52$

3.1. Estratégia de Pesquisa

3.2. Tipo de Pesquisa $\quad 54$

3.3. Fases da Pesquisa $\quad 55$

3.4. Universo da Pesquisa $\quad 57$

3.5. Seleção de Sujeitos 60

3.6. Coleta de Dados 61

3.6.1. Pesquisa Bibliográfica 61

3.6.2. Pesquisa Documental e Telematizada 61

3.6.3. Pesquisa de Campo 62 
3.7. Tratamento dos Dados $\quad 67$

3.8. Limitações do Método 68

4 Análise e Discussão dos Resultados $\quad 69$

4.1. O Entendimento sobre a Qualificação Profissional 69

4.2. Categorização dos temas $\quad 71$

4.2.1. A percepção da qualificação profissional dos trabalhadores 72

4.2.2. O treinamento para a qualificação profissional 80

4.2.3. Crescimento e oportunidades da indústria de óleo, gás e energia na região $\begin{array}{ll}\text { Nordeste } & 88\end{array}$

4.2.4. Alinhamento e parceria para a melhoria da qualificação 92

5 Conclusão e Considerações Finais 99

$\begin{array}{ll}\text { Referências Bibliográficas } & 104\end{array}$

$\begin{array}{ll}\text { Bibliografia } & 111\end{array}$

$\begin{array}{ll}\text { Anexos } & 114\end{array}$

Anexo I Definição dos Termos e Siglas 114 


\section{Lista de figuras}

Figura 1 - Gráfico dos níveis de investimentos da Petrobras 14

Figura 2 - Investimentos previstos pela Petrobras - Período 2009-2013 15

Figura 3 - Evolução da produção total da Petrobras e metas de crescimento 16

$\begin{array}{ll}\text { Figura } 4 \text { - Prominp } & 18\end{array}$

Figura 5 - Demanda de profissionais qualificados $\quad 19$

Figura 6 - Educação, instrução e treinamento e o desenvolvimento integral do indivíduo 42

Figura 7 - Ações de aprendizagem em ambientes organizacionais $\quad 44$

Figura 8 - Fases da Pesquisa 55 


\section{Lista de tabelas}

Tabela 1 - Investimentos da Petrobras por período 14

Tabela 2 - Metas do PNQP 20

Tabela 3 - Demanda e total de vagas por Estado 20 


\section{Lista de quadros}

Quadro 1 - Conceitos e definições das ações de aprendizagem

45

Quadro 2 - Descrição dos empreendimentos e projetos estudados

58

Quadro 3 - Sites consultados na internet

62

Quadro 4 - Sujeitos selecionados para pesquisa qualitativa 